\title{
Rapid Test Method for Multi-Beam Profile of Phased Array An- tennas
}

\author{
Qingchun Luo ${ }^{1}$, Yantao zhou ${ }^{2,}$, Yihong $\mathrm{Qi}^{3}, \mathrm{Pu} \mathrm{Ye}^{4}$, Francesco de Paulis ${ }^{5}$, Lie Liu ${ }^{6}$ \\ 1 Hunan University, CN; $1733835492 @ q q . c o m$ \\ 2 Hunan University, CN; yantao_z@hnu.edu.cn \\ 3 Pengcheng Laboratory, CN; yihong.qi@generaltest.com \\ 4 Shenzhen General Test System Inc., CN; pu.ye@generaltest.com \\ 5 University of L'Aquila, 67100 L'Aquila, Italy; francesco.depaulis@univaq.it \\ 6 Shenzhen General Test System Inc., CN; lie.liu@generaltest.com \\ * Correspondence: yantao_z@hnu.edu.cn, CN; Tel.: +86 13973117637
}

\begin{abstract}
The testing requirements of the active phased array antennas are very different from those of traditional passive antennas, due to its beam steering capability. Usually, each beam profile of the active phased array needs a separate radiation pattern test, which makes the overall testing time extremely long. Thus the traditional antenna test method can no longer meet the efficiency and cost requirements of new active phased array antennas test. In this paper, a fast test method tailored for phased array antennas is proposed that offers significantly reduced testing time at the expense of slight sacrifice of the accuracy. Using the simulated element pattern in array and ideal port excitation, the beam profile in any direction can be predicted by testing only a certain beam profile. Through theoretical derivation and experiments, the effectiveness of the method is verified, and the testing efficiency of the phased array antenna is demonstrated to be improved by ten times or even more.
\end{abstract}

Keywords: Multi-beam profile fast test method, Phased array antenna, Source reconstruction, Antenna test method

\section{Introduction}

Due to its high gain and adjustable beam pointing characteristics, phased array antennas are widely used in mobile and satellite communications, radars (for automotive sensing and unmanned aerial vehicles), as well as in military and meteorological applications [1-6].

Advances in materials (for example, GaN etc.) and manufacturing processes (AIP) have improved phased array antenna performance and reduced costs dramatically, allowing it to be widely used [7], which result in high volume production and demand in testing of phased array antennas. Unlike conventional antennas, large phased array antennas are characterized by thousands of beam profiles and each one needs to be tested individually. It becomes a great challenge to the existing antenna test systems since the testing time will drastically increase compared with that of conventional antennas [8,9]. Traditional test methods are incompetent to meet the demand of mass production test. Methods have been proposed to improve the testing efficiency by reducing the number of profiles tested and the number of sampling points on individual profile [10-12]. However, the testing time is not significantly reduced, but the error introduced is quite large. Therefore, fast testing of multi-beam profiles is a critical problem that needs to be solved for the mass production of phased array antennas.

In this paper, a physical model-based source reconstruction method [13-16] is proposed to reduce testing time of multi beam profiles of phased-array antennas. The radiated field of a phased-array antenna is considered to be an iterative addition of the radiated field of each element constituting the array [17]. The proposed method uses the 
radiated field and the port excitation information of a single element to invert the aperture field excitation coefficient matrix of the phased-array antenna by sampling the pattern of individual beam profile. Using the obtained aperture field excitation coefficients, the known pattern of the array element, and the port excitation associated to any beam profile, the far-field pattern of the beam profile is predicted. This method is based on the actual physical model of the phased array, and it utilizes the information of the radiation pattern of the element among the actual array, and thus it has higher accuracy compared to the conventional source reconstruction methods.

In this paper, the predicted beam profile patterns are compared with the measured profile patterns to validate the effectiveness of the method. For a phased array antenna in which dozens or even hundreds of beam profiles need to be tested, the conventional method can only test each beam profile individually. However, by using the method proposed in this paper, only one beam profile test is needed to obtain the excitation coefficient of the phased array, and then the far-field radiation pattern of arbitrary beam profile can be predicted with acceptable accuracy, which greatly shortens the testing time of all required beam profiles to be tested for a multibeam phased array antenna.

\section{Theory and Methods}

An array of $\mathrm{N}$ elements, as the one shown in Figure. 1 is characterized by the far-field radiation pattern $\mathrm{E}(\theta, \phi)$ expressed in (1)

$$
E(\theta, \phi)=\sum_{i=1}^{N} I^{\prime}{ }_{i} e^{j \varphi_{I_{i}}} \tilde{f}_{i}(\theta, \phi) e^{j k\left(r_{i} \cdot \hat{r}_{o}\right)}
$$

Where $\tilde{f}_{i}(\theta, \phi)$ is the far-field radiation pattern of the $i$-th element within the array, i.e., the far-field radiation pattern in the presence of all the other surrounding elements, $I^{\prime}{ }_{i} e^{j \varphi_{I_{i}}^{\prime}}$ is the aperture field excitation of the $i$-th element, $\overrightarrow{r_{o}(\theta, \phi)}$ represents the vector from the coordinate origin to the test point, $\overrightarrow{r_{l}(\theta, \phi)}$ denotes the vector from the position of the $i$-th element to the test point. Basically, (1) implies that the far-field radiation is obtained as combination (sum) of the radiation pattern $\tilde{f}_{i}(\theta, \phi)$ of all elements. The coupling effect among the elements are taken into account by the far-field radiation term $\tilde{f}_{i}(\theta, \phi)$, whereas other non-ideal factors such as excitation and matching of elements, manufacturing error, and $\mathrm{T} / \mathrm{R}$ component differences are considered by the aperture field

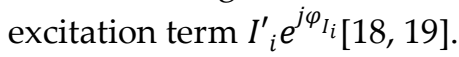

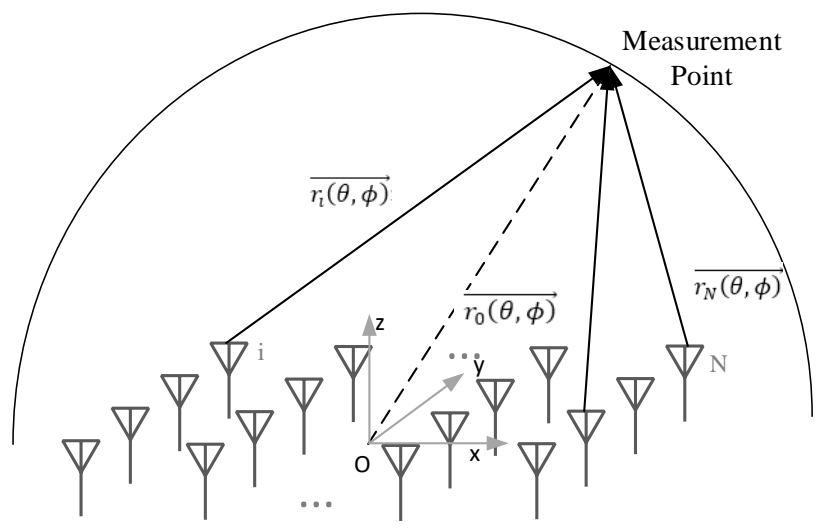

Figure. 1. Schematic diagram of the array with total $N$ number of elements.

For a particular beam profile, an ideal excitation I on all element ports can be defined as in (2), where $\mathrm{N}$ is the total number of elements. Whereas I' in (3) represents the excitation vector at the radiating aperture for a specific beam profile, and it is obtained from I using the aperture field excitation coefficient matrix $C$ for each element. This excitation 
coefficient matrix $C$ is well-defined for a specific phased array DUT since it is determined by the port matching, element manufacturing error, $\mathrm{T} / \mathrm{R}$ component differences, etc.

$$
\begin{gathered}
I=\left[\begin{array}{c}
I_{1} e^{j \varphi_{I_{1}}} \\
I_{2} e^{j \varphi_{I_{2}}} \\
\vdots \\
I_{N} e^{j \varphi_{I_{N}}}
\end{array}\right] \\
I^{\prime}=\left[\begin{array}{c}
I_{1}^{\prime} e^{j \varphi_{I_{1}}^{\prime}} \\
I_{2}^{\prime} e^{j \varphi_{I_{2}}^{\prime}} \\
\vdots \\
I_{N}^{\prime} e^{j \varphi_{I_{N}}^{\prime}}
\end{array}\right]=I \cdot C=\left[\begin{array}{c}
I_{1} e^{j \varphi_{I_{1}}} \\
I_{2} e^{j \varphi_{I_{2}}} \\
\vdots \\
I_{N} e^{j \varphi_{I_{N}}}
\end{array}\right] \cdot\left[\begin{array}{c}
C_{1} \\
C_{2} \\
\vdots \\
C_{N}
\end{array}\right]
\end{gathered}
$$

A radiation pattern vector can also be defined, according to (4). By using (3) and (4), (1) can be expressed as in (5). The objective is to solve (5) to obtain the aperture field excitation coefficient matrix $C$ of the phased array antenna, which is then used for the radiation pattern prediction of the remaining beam profiles. This goal is achieved by knowing the amplitude and phase data of the radiated field $E(\theta, \phi)$. By performing far-field measurements on the phased-array antenna, $M$ far-field test data can be obtained, $E_{j}(\mathrm{j}=1,2, \ldots$, $\mathrm{M})$, where, $E_{j}=E\left(\theta_{j}, \phi_{j}\right)$, and $M \geq N$; with such data available, the linear equation system in (6) can be defined

$$
\begin{gathered}
F_{o}=\left[\begin{array}{c}
\tilde{f}_{1}(\theta, \phi) e^{j k\left(r_{1} \cdot \hat{r}_{o}\right)} \\
\tilde{f}_{2}(\theta, \phi) e^{j k\left(r_{2} \cdot \hat{r}_{o}\right)} \\
\vdots \\
\tilde{f}_{N}(\theta, \phi) e^{j k\left(r_{N} \cdot \hat{r}_{o}\right)}
\end{array}\right] \\
E(\theta, \phi)=F_{o}^{T}(I \cdot C) \\
{\left[\begin{array}{c}
E_{1} \\
E_{2} \\
\vdots \\
E_{M}
\end{array}\right]=\left[\begin{array}{cc}
\tilde{f}_{1}\left(\theta_{1}, \phi_{1}\right) e^{j k\left(r_{11} \cdot \hat{r}_{01}\right)} & \ldots \tilde{f}_{N}\left(\theta_{1}, \phi_{1}\right) e^{j k\left(r_{N 1} \cdot \hat{r}_{01}\right)} \\
\tilde{f}_{1}\left(\theta_{2}, \phi_{2}\right) e^{j k\left(r_{12} \cdot \hat{r}_{02}\right)} & \ldots \tilde{f}_{N}\left(\theta_{1}, \phi_{1}\right) e^{j k\left(r_{N 1} \cdot \hat{r}_{01}\right)} \\
\vdots & \vdots \\
\tilde{f}_{N}\left(\theta_{M}, \phi_{M}\right) e^{j k\left(r_{1 M} \cdot \hat{r}_{o M}\right)} \cdots & \tilde{f}_{N}\left(\theta_{1}, \phi_{1}\right) e^{j k\left(r_{N 1} \cdot \hat{r}_{01}\right)} \\
\left.\tilde{f}_{1}\right) e^{j k\left(r_{N 1} \cdot \hat{r}_{01}\right)}
\end{array}\right] \cdot\left[\begin{array}{c}
I_{1}^{\prime} e^{j \varphi_{I_{1}}^{\prime}} \\
I_{2}^{\prime} e^{j \varphi_{I_{2}}^{\prime}} \\
\vdots \\
I_{N}^{\prime} e^{j \varphi_{I_{N}}^{\prime}}
\end{array}\right]}
\end{gathered}
$$

Where $\hat{r}_{o j}(j=1,2, \cdots, M)$ represents the unit vector from the coordinate origin to the $j$ th test point, $r_{i j}(i=1,2, \cdots, N ; j=1,2, \cdots, M)$ represents the vector between the $i$-th element and the $\mathrm{j}$-th test point, $\tilde{f}_{i}\left(\theta_{j}, \phi_{j}\right)$ represents the radiation field of the $\mathrm{i}$-th element at the $\mathrm{j}$-th measurement point $\left(\theta_{j}, \phi_{j}\right)$,

In (6) the vector $E$ and the matrix $F$ can be defined according to (7) and (8), respectively. Thus the compact form in (9) is obtained.. Both side of (9) are multiplied by the term $\left(F^{T} F\right)^{-1} F^{T}$ to obtain the solution $I^{\prime}$ in (10) of the excitations at the element apertures.

$$
\begin{gathered}
E=\left[\begin{array}{c}
E_{1} \\
E_{2} \\
\vdots \\
E_{M}
\end{array}\right] \\
F=\left[\begin{array}{ccc}
\tilde{f}_{1}\left(\theta_{1}, \phi_{1}\right) e^{j k\left(r_{11} \cdot \hat{r}_{01}\right)} & \ldots & \tilde{f}_{N}\left(\theta_{1}, \phi_{1}\right) e^{j k\left(r_{N 1} \cdot \hat{r}_{o 1}\right)} \\
\tilde{f}_{1}\left(\theta_{2}, \phi_{2}\right) e^{j k\left(r_{12} \cdot \hat{r}_{O 2}\right)} & \cdots & \tilde{f}_{N}\left(\theta_{2}, \phi_{2}\right) e^{j k\left(r_{N 2} \cdot \hat{r}_{O 2}\right)} \\
\vdots & \vdots \\
\tilde{f}_{1}\left(\theta_{M}, \phi_{M}\right) e^{j k\left(r_{1 M} \cdot \hat{r}_{O M}\right)} \cdots & \tilde{f}_{N}\left(\theta_{M}, \phi_{M}\right) e^{j k\left(r_{N M} \cdot \hat{r}_{o M}\right)}
\end{array}\right] \\
E=F I^{\prime}=F(I \cdot C) \\
\left(F^{T} F\right)^{-1} F^{T} E=\left(F^{T} F\right)^{-1} F^{T} F I^{\prime}=I^{\prime}=(I \cdot C)
\end{gathered}
$$




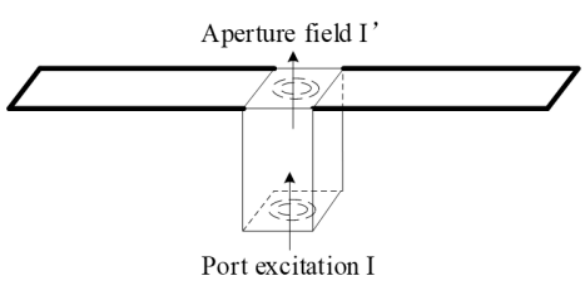

Figure. 2. Schematic diagram of port excitation and aperture field excitation of phased array element

So far, through the $M$ far-field measurement points obtained by testing the $N$-element array antenna, and the through the radiation pattern $F$ of the array elements obtained by simulation or actual measurement, the aperture field excitation $I^{\prime}$ of the element can be obtained. When $M=N$, the number of equations is equal to the number of unknowns to be solved, and the aperture field excitation $I^{\prime}$ can be obtained by simply solving a linear equation system of equations. However, in the more general case of $\mathrm{M}>\mathrm{N}$, the number of equations is greater than the number of unknowns, and the aperture field excitation $I^{\prime}$ can be obtained by applying the least square method. Since the ideal element port excitation I for a particular beam profile is assumed to be known (theoretical value), the aperture field excitation coefficient matrix $C$ can be readily obtained. With the coefficient $C$, the radiation pattern $E_{x}$ for any beam profile can be obtained by applying (11).

$$
E_{x}=F I_{x}^{\prime}=F\left(I_{x} \cdot C\right)
$$

In this way, the radiation pattern of all beam profiles of the phased array can be readily predicted, thus relying only on one single radiation pattern measurement. This method becomes obviously more effective for large antennas characterized with a large number of elements and beam profiles.

In order to verify the feasibility of the proposed method, an active phased array antenna with adjustable beam profiles is tested in the far-field anechoic chamber.

The validation steps are as follows,

1. Install the phased-array antenna on the test system turntable and adjust the center of the antenna to coincide with the center of the rotation system,

2. Use the beam controller to adjust the phased array antenna to a certain main beam orientation $\left(\theta_{0}, \varphi_{0}\right)$ and test the radiation pattern $E_{0}$,

3. Obtain the element port excitation file I of the selected beam profile $\left(\theta_{0}, \varphi_{0}\right)$,

4. Obtain the simulation radiation pattern $\mathrm{F}$ of all the elements among the array with the coupling factor taken into account,

5. Use (10) to calculate the aperture field coupling coefficient $C$ of this antenna,

6. Apply the element port excitation $I_{x}$ in (11) of the beam profile to be predicted $\left(\theta_{x}\right.$, $\left.\varphi_{x}\right)$,

7. Calculate the radiation pattern $E_{x}$ of the beam profile to be predicted $\left(\theta_{x}, \varphi_{x}\right)$ using (11),

8. Adjust the phased array antenna to the beam profile to be predicted $\left(\theta_{x}, \varphi_{x}\right)$ by the wave controller, and measure the actual radiation pattern of this beam profile,

9. Compare $E_{x}$ and $\widetilde{E_{x}}$.

\section{Results and Discussions}

A 64-element phased-array antenna was used to test and validate the proposed fast testing methodology in a spherical test range chamber with a $2.5 \mathrm{~m}$ measurement distance, as shown in Figure 3. The phased-array antenna array size is about $80^{*} 80 \mathrm{~mm}$ and the test frequency is $25 \mathrm{GHz}$, thus the distance can meet the far-field test conditions.

The test radiation pattern at Step 2 is selected at $\left(\theta_{0}, \varphi_{0}\right)=(0,0)$, with a sampling range of $\varphi=0 \sim 180^{\circ}, \theta=-90^{\circ} \sim 90^{\circ}$, and a sampling step of $2^{\circ}$. Based on the simulated radiation pattern obtained at Step 4 and the ideal port excitation $I$ of all the elements within the 
array, the aperture field excitation coefficient $C$ of the elements is obtained by using (10), according to Step 5 . Once $C$ is known, and by using (11), the radiation patterns of three different beam profiles, $(\theta, \varphi)$ at $(15,0),(30,0),(60,0)$ are predicted and compared with the measured values, as shown in Figure 4. A detailed analysis of the first side lobes amplitude is carried out and the errors between the measured and predicted side lobe amplitudes are reported in Tables 1-3 for the three considered cases. Also, the error in the main lobe $3 \mathrm{~dB}$ beamwidth and in the cross-polarization level are quantified.
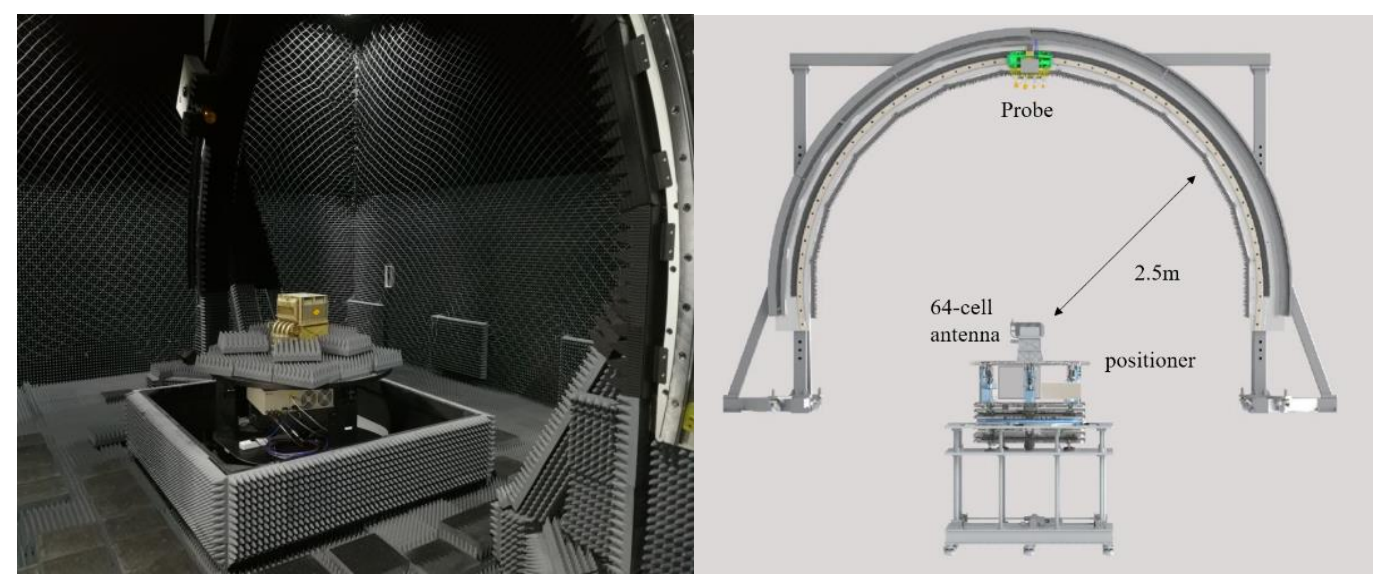

Figure. 3. The 64-element Phased Array Antenna in The Spherical Range Measurement System

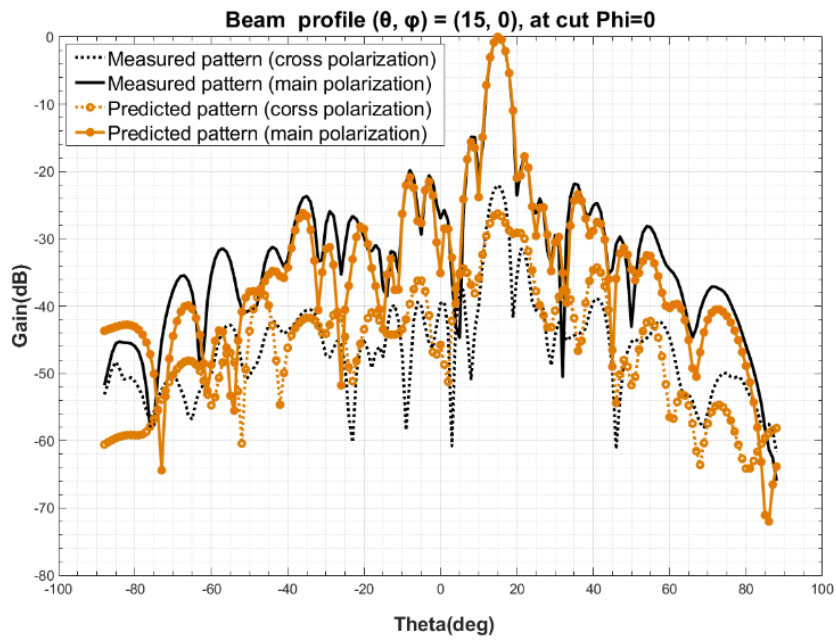

Figure. 4. Comparison of predicted and measured radiation pattern of beam profile $(15,0)$, at cut Phi $=0$

Table 1. Measured vs. Predicted Pattern. of Beam at $(\theta, \varphi)=(15,0)$

\begin{tabular}{|l|r|r|r|r|}
\hline & $\begin{array}{c}\text { 3dB Beam } \\
\text { Width }\left(^{\circ}\right)\end{array}$ & $\begin{array}{c}\text { First left } \\
\text { side lobe } \\
\text { peak }(\mathrm{dB})\end{array}$ & $\begin{array}{c}\text { First right } \\
\text { side lobe } \\
\text { peak }(\mathrm{dB})\end{array}$ & $\begin{array}{c}\text { Cross-pol. } \\
(\mathrm{dB})^{1}\end{array}$ \\
\hline Measured & 4.18 & -14.58 & -18.19 & 19.568 \\
\hline Predicted & 4.25 & -15.33 & -18.17 & 19.685 \\
\hline Deviation & 0.07 & -0.75 & 0.02 & 0.117 \\
\hline
\end{tabular}

${ }^{1}$ The cross-polarization is the maximum difference between the main polarization and the crosspolarization within a $3 \mathrm{~dB}$ beamwidth. 


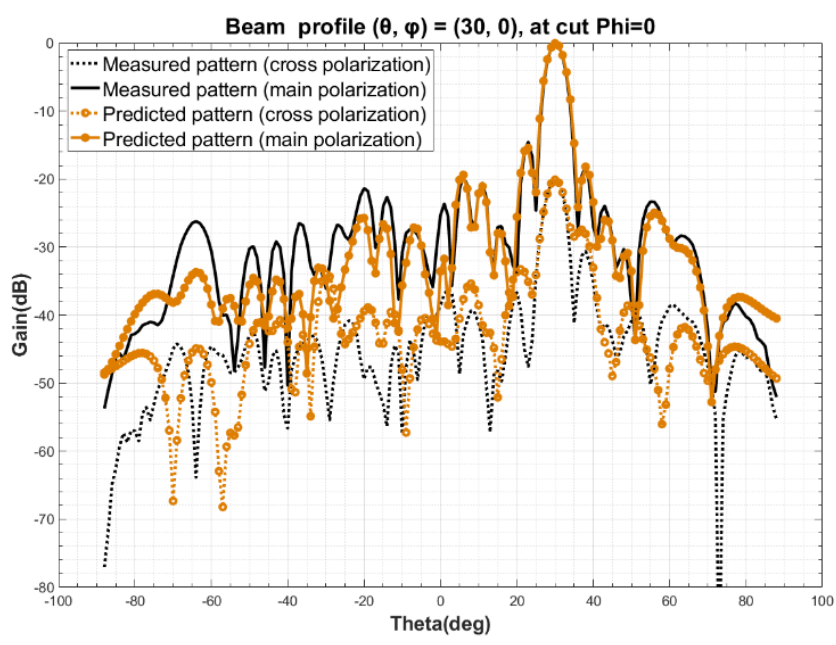

Figure. 5. Comparison of predicted and measured radiation pattern of beam profile $(30,0)$, at cut Phi $=0$

Table 2. Measured vs. Predicted Pattern. of Beam at $(\theta, \varphi)=(30,0)$

\begin{tabular}{|l|r|r|r|r|}
\hline & $\begin{array}{c}\text { 3dB Beam } \\
\text { Width }\left(^{\circ}\right)\end{array}$ & $\begin{array}{c}\text { First left } \\
\text { side lobe } \\
\text { peak }(\mathrm{dB})\end{array}$ & $\begin{array}{c}\text { First right } \\
\text { side lobe } \\
\text { peak }(\mathrm{dB})\end{array}$ & $\begin{array}{c}\text { Cross-pol. } \\
(\mathrm{dB})^{1}\end{array}$ \\
\hline Measured & 4.54 & -14.94 & -17.44 & -21.752 \\
\hline Predicted & 4.66 & -15.55 & -17.76 & -24.461 \\
\hline Deviation & 0.12 & 0.61 & 0.32 & 2.709 \\
\hline
\end{tabular}

${ }^{1}$ The cross-polarization is the maximum difference between the main polarization and the crosspolarization within a $3 \mathrm{~dB}$ beamwidth.

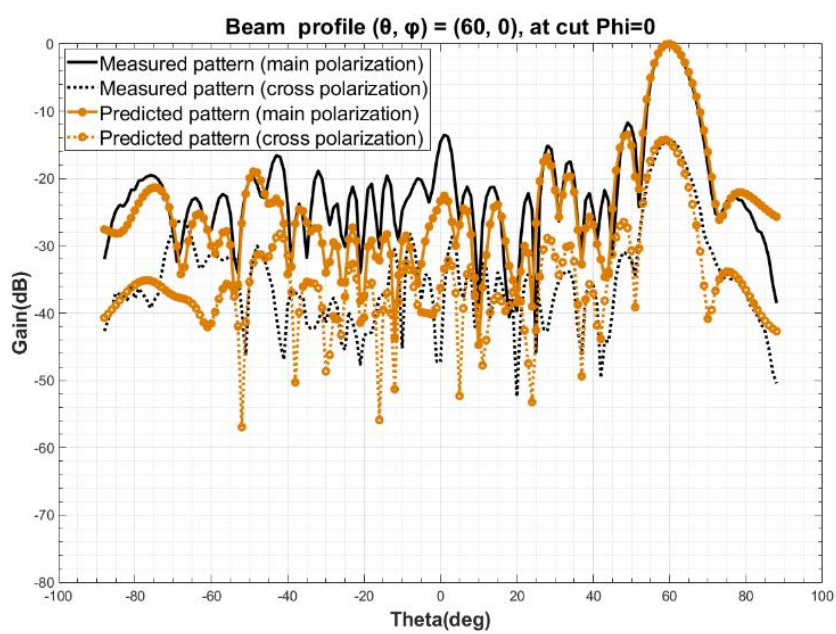

Figure. 6. Comparison of predicted and measured radiation pattern of beam profile $(60,0)$, at cut Phi $=0$

Table 3 Measured vs. Predicted Pattern. of Beam at $(\theta, \varphi)=(60,0)$

\begin{tabular}{|l|r|r|r|r|}
\hline & $\begin{array}{c}\text { 3dB Beam } \\
\text { Width }\left(^{\circ}\right)\end{array}$ & $\begin{array}{c}\text { First left } \\
\text { side lobe } \\
\text { peak }(\mathrm{dB})\end{array}$ & $\begin{array}{c}\text { First right } \\
\text { side lobe } \\
\text { peak }(\mathrm{dB})\end{array}$ & $\begin{array}{c}\text { Cross-pol. } \\
(\mathrm{dB})^{1}\end{array}$ \\
\hline Measured & 7.84 & -11.79 & -22.62 & -14.012 \\
\hline Predicted & 8.11 & -13.21 & -22.07 & -12.952 \\
\hline Deviation & 0.27 & 1.42 & 0.55 & -1.06 \\
\hline
\end{tabular}


${ }_{1}^{1}$ The cross-polarization is the maximum difference between the main polarization and the crosspolarization within a $3 \mathrm{~dB}$ beamwidth.

\section{Discussion}

The comparison of the data above in Figures. 4-6 and in Table 1-3 shows that the predicted and measured radiation patterns agree well within the range of both the primary and secondary lobes at different beam profile $(\theta, \varphi)$, even for large scan angles, i.e. $(\theta, \varphi)=(60,0)$. The deviation between the predicted and measured is less than $0.75 \mathrm{~dB}$ at the side lobe, which is over $15 \mathrm{~dB}$ lower than the main lobe, and less than $\pm 1.5 \mathrm{~dB}$ at a crosspolarization ratio about $-25 \mathrm{~dB}$. The difference between the predicted and measured values of the side lobes and in the cross-polarization level meets the general uncertainty requirement of phased array antenna test [20-23].

The standard method for testing a 64-element phased-array antenna considered for validation in this work would require to sweep the main beam within the following range, $\theta=0^{\circ} \sim 60^{\circ}$ and $\varphi=0^{\circ} \sim 360^{\circ}$, with $15^{\circ}$ angular interval. Therefore, the total number of beam profiles needs to be tested is 49 . The test time for one beam profile is about 30 minutes. The source reconstruction method, except for the calculation of the excitation coefficient $C$ of the aperture field, which takes about 1 minute, uses about $30 \mathrm{~min}$ for predicting the full radiation pattern. A comparison of the efficiency of the fast test method and the conventional method is shown in Table 4.

Table 4 Efficiency Comparison

\begin{tabular}{|l|c|c|}
\hline & $\begin{array}{c}\text { Source } \\
\text { Reconstruction } \\
\text { Method }\end{array}$ & $\begin{array}{c}\text { Traditional } \\
\text { Method }\end{array}$ \\
\hline Coefficient C calculating [min] & 1 & 0 \\
\hline First beam profile measurement [min] & 30 & 30 \\
\hline 48 beam profiles measurement [min] & 48 & 1440 \\
\hline Total duration [min] & 79 & 1470 \\
\hline
\end{tabular}

the proposed source reconstructed method can greatly improve the testing efficiency and minimize the testing time and cost for multi-beam profiles of phased array antennas. Beside the good agreement and acceptable errors in the prediction of the first side lobes, larger amplitude differences are found for far side lobes. Such error is presumed to be caused by the combined effect of the following factors which makes the aperture field excitation coefficient matrix $\mathrm{C}$ calculated by a single beam profile different from the real one: the difference between the simulation and the actual radiation pattern of the array elements, the installation position deviation of the antenna, the uncertainties of the test system and of the nominal excitation values $I x$ of each element.

\section{Conclusions}

In this paper, a multi-beam profile fast test method for phased array antennas based on source reconstruction algorithm is proposed. Through theoretical derivation and experimental verification, it is found that the fast test method proposed in this paper can obtain the aperture field excitation coefficient of phased array antenna element through the radiation pattern measurement at a single beam profile, and then predict the radiation pattern of arbitrary beam profile. The predicted radiation pattern of the beam matches very well with the measured one on the main lobe, although a bit bad on the side lobes. This method avoids testing the beam profiles one by one, thus greatly improving the testing efficiency of phased array antennas.

A further improvement of the proposed method will be worked out in the future by analyzing the accuracy when more than one measured beam pattern is used to find more accurate solutions for the excitation coefficient matrix C. Further research will also be conducted for the scenarios where the element radiation patterns within the array are not known. 


\section{References}

1. K. Anim; J.N. Lee and Y.-B. Jung. High-Gain Millimeter-Wave Patch Array Antenna for Unmanned Aerial Vehicle Application. Sensors 2021, vol. 21, no. 11, p. 3914. [Online]. Available: https://www.mdpi.com/1424-8220/21/11/3914.

2. Li J.; Wang J H.; Liu Y G; et al. The Application And Prospect of Phased Array Antenna in Satellite Communication [J]. Radio Engineering 2019, 049(012): 1076-84.

3. Zhou K.Q.; Wang H.C. Joint Ship and Artillery Snti-missile Technology based on Phased Array Radar [J]. Firepower and Command Control 2019, v.44, No.291(06): 161-5.

4. Zhang Z.Q.; Liu L P. Preliminary Application of Phased array Technology in Weather Radar [J]. Highland Meteorology 2011, 30(4): 1102-7.

5. Srivastava; R.C. Applications of weather radar systems: A guide to uses of radar data in meteorology and hydrology By C.G. Collier, Ellis Harwood. Journal of Hydrology 1989, 130.1-4(1992):408-409.

6. J. S. Herd; M. D. Conway. The Evolution to Modern Phased Array Architectures. Proceedings of the IEEE 2016, vol. 104, no. 3, pp. 519-529.

7. Lee S.; H. Kim K.J.; Park S.; et al. Implementation of High Efficiency Amplifier with GaN Doherty Structure for LTE-Advanced Active Phased Arrays Antenna System[J]. Proceedings of Symposium of the Korean Institute of communications and Information Sciences. 2014.

8. Yihong. Qi; et al. 5G Over-the-Air Measurement Challenges: Overview. IEEE Transactions on Electromagnetic Compatibility 2017.

9. G. Ruggerini; P. G. Nicolaci; and G. Toso. A Ku-Band Magnified Active Tx/Rx Multibeam Antenna Based on a Discrete Constrained Lens, Electronics 2021, vol. 10, no. 22, p. 2824. [Online]. Available: https://www.mdpi.com/2079-9292/10/22/2824.

10. Z. Wang; C. Pang; Y. Li and X. Wang. A Method for Radiation Pattern Reconstruction of Phased-Array Antenna. IEEE Antennas and Wireless Propagation Letters 2020, vol. 19, no. 1, pp. 168-172, doi: 10.1109/lawp.2019.2956761.

11. N. R. Leonor; R. F. S. Caldeirinha; M. G. Sánchez; and T. R. Fernandes. A Three-Dimensional Directive Antenna Pattern Interpolation Method. IEEE Antennas and Wireless Propagation Letters 2015.

12. T. Petrita and A. Ignea. A new method for interpolation of 3D antenna pattern from $2 \mathrm{D}$ plane patterns. International Symposium on Electronics \& Telecommunications 2013.

13. Z. Ji; D. Pommerenke and J. Fan. Determining Equivalent Dipoles Using a Hybrid Source-Reconstruction Method for Characterizing Emissions From Integrated Circuits. IEEE Transactions on Electromagnetic Compatibility 2017,vol. 59, no. 2, pp. 567-575.

14. Q. Huang and J. Fan. Machine Learning Based Source Reconstruction for RF Desense. IEEE Transactions on Electromagnetic Compatibility 2018, vol. PP, no. 6, pp. 1-8.

15. Z. Ji and J. Fan. Source Reconstruction for IC Radiated Emissions Based on Magnitude-Only Near-Field Scanning. IEEE Transactions on Electromagnetic Compatibility 2017, vol. PP, no. 99, pp. 1-10.

16. H. Rezaei; J. Meiguni; M. Soerensen; J. Fan, and D. Pommerenke. Source Reconstruction in Near Field Scanning using Inverse MoM for RFI Application. IEEE International Symposium on Electromagnetic Compatibility and Signal E Power Integrity.

17. D. F. Kelley and W. L. Stutzman. Array antenna pattern modeling methods that include mutual coupling effects. IEEE Transactions on Antennas \& Propagation 1993, vol. 41, no. 12, pp. 1625-1632.

18. D. M. Pozar. A relation between the active input impedance and the active element pattern of a phased array. IEEE Transactions on Antennas \& Propagation 2003, vol. 51, no. 9, pp. 2486-2489.

19. D. M. Pozar. The active element pattern. IEEE Transactions on Antennas \& Propagation 1994, vol. 42, no. 8, pp. 1176-1178.

20. Haselwander, W.; et al. Measurement on an Active Phased Array Antenna on a Near-Field Range and an Anechoic Far-Field Chamber. 2001 31st European Microwave Conference IEEE 2007.

21. Gao H.; Fan W.; Wang W.; et al. On Uncertainty Investigation of mmWave Phased-Array Element Control With an All-On Method[J]. IEEE Antennas and Wireless Propagation Letters 2020, PP(99):1-1.

22. Salas-Natera; Miguel A. and R. RodrEz-Osorio. Analytical Evaluation of Uncertainty on Active Antenna Arrays. IEEE Transactions on Aerospace \& Electronic Systems. 48.3(2012):1903-1913..

23. Wang, J.; et al. Study of wide-angle scanning phased array antenna measurement. Journal of Xidian University 2015. 